\title{
Nematic resonance in the Raman response of iron-based superconductors
}

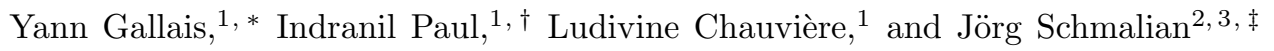 \\ ${ }^{1}$ Laboratoire Matériaux et Phénomènes Quantiques, UMR 7162 CNRS, \\ Université Paris Diderot, Bat. Condorcet 75205 Paris Cedex 13, France \\ ${ }^{2}$ Institute for Theory of Condensed Matter, Karlsruhe Institute of Technology (KIT), 76131 Karlsruhe, Germany \\ ${ }^{3}$ Institute for Solid State Physics, Karlsruhe Institute of Technology (KIT), 76131 Karlsruhe, Germany
}

(Dated: October 10, 2018)

In a fully-gapped superconductor the electronic Raman response has a pair-breaking peak at twice the superconducting gap $\Delta$, if the Bogoliubov excitations are uncorrelated. Motivated by the iron based superconductors, we study how this peak is modified if the superconducting phase hosts a nematic-structural quantum critical point. We show that, upon approaching this point by tuning, e.g., doping, the growth of nematic correlations between the quasiparticles transforms the pair-breaking peak into a nematic resonance. The mode energy is below $2 \Delta$, and stays finite at the quantum critical point, where its spectral weight is sharply enhanced. The latter is consistent with recent experiments on electron-doped iron based superconductors and provides direct evidence of nematic correlations in their superconducting phases.

Introduction.- Nematicity in correlated metals implies the propensity of the interacting electron fluid to break rotational symmetry without necessarily breaking translation symmetry [1]. It is at the heart of the phase competition in iron based superconductors (Fe SC) [2], leading to a lattice softening 3 [5, transport and magnetic anisotropies [6-10, signatures in optical [11, 12], single particle [12 15 and Raman [16 spectroscopies in the normal state. In the latter, the critical nematic fluctuations in the tetragonal phase appear in the $B_{1 g}$ Raman response as a low energy quasi-elastic peak.

While nematicity in the normal state of Fe SC has been studied extensively, it is unclear whether nematic fluctuations couple to low energy charge carriers in the superconducting state. In fact, even the existence and detailed nature of the nematic fluctuations below $T_{c}$ is not established either experimentally or theoretically.

In this Letter we demonstrate that, near a nematic quantum critical point (QCP) which is well inside a fullygapped superconducting phase [17, 18, the growth of nematic correlation transforms the standard pair-breaking peak in the Raman $B_{1 g}$ channel into a new nematic resonance mode at finite frequency. In a clean system the resonance is a sharp structure below $2 \Delta$ of the electron pockets of $\mathrm{Fe} \mathrm{SC}$, and its spectral weight increases strongly near the QCP. The latter, which is a key feature of the theory, and which cannot be understood within a pair-breaking peak scenario, is consistent with existing Raman scattering measurements in Co doped Ba-122 [19, 20] and Na-111 [21] systems. This gives very strong evidence for this new mode, and, in turn, reveals that there is a substantial coupling between Bogoliubov quasiparticles and nematic fluctuations.

When compared to the normal state behavior, the resonance in the superconducting phase can be understood as the consequence of the opening of the gap, which shifts spectral weight to higher frequencies, thereby transforming the quasi-elastic peak into a resonance. It is caused exclusively by electronic contributions, while renormalizations due to the lattice are dynamically screened and are thus suppressed. This leads to the interesting result that the Raman nematic resonance energy remains finite even at a nematic QCP.

The nematic resonance is analogous to the spinresonance, observed in neutron scattering experiments in Fe SC and cuprates superconductors 22 27. In fact, there is a close formal analogy of our theory to the one for the spin-resonance mode in superconductors with sign changing gap near a magnetic instability [28, 29]. In both cases a singular behavior of the imaginary part of a bare response function, caused by the superconducting coherence factors, is amplified by the vicinity to a QCP. Important differences are that the singularity in our case is not related to a sign change of the gap as small momentum excitations are probed. For the same reason the Raman resonance is more sensitive to nodes of the gap.

Nematic resonance.- In the following we develop a theory for the nematic resonance, and we argue that it is intimately related to the quasi-elastic peak with diverging spectral weight experimentally observed in the normal state $B_{1 g}$ Raman response of electron doped Fe SC [16, 21]. The $B_{1 g}$ quasi-elastic peak is a signature of the presence of critical fluctuations of the the nematic operator

$$
\rho_{B_{1 g}}(\mathbf{q})=\frac{1}{N} \sum_{\mathbf{k} \sigma} \gamma_{B_{1 g}}(\mathbf{k}) \psi_{\mathbf{k}+\frac{\mathbf{q}}{2}}^{\dagger} \psi_{\mathbf{k}-\frac{\mathbf{q}}{2}}
$$

whose susceptibility can be accessed in Raman scattering experiments in $B_{1 g}$ geometry [30]. Here $\gamma_{B_{1 g}}(\mathbf{k})$ transforms as $k_{x}^{2}-k_{y}^{2}$ under the point group operations. Note that, Raman experiments thus imply that the nematic instability has a $d$-wave Pomeranchuk component. We take this as our starting point, and write a phenomenological interaction $\mathcal{H}_{I}=-g / 2 \sum_{\mathbf{q}} \rho_{B_{1 g}}(\mathbf{q}) \rho_{B_{1 g}}(-\mathbf{q})$, with $g>0$, that can be tuned to study the Pomeranchuknematic QCP. The microscopic origin of $g$, which can 
be due to spin or bond-current [17, 31,34] or orbital [35, 37-39] or charge [40] fluctuations, is not relevant for the following discussion. Note that, fluctuations associated with a Pomeranchuk instability are, by definition, intra-band terms. Furthermore, it is thought that the $B_{1 g}$ Raman response of electron-doped Fe SC is governed by the electron pockets 441, 42. Consequently, to simplify the discussion, by $\left(\psi_{\mathbf{k}}^{\dagger}, \psi_{\mathbf{k}}\right)$ we imply the fermions describing the electron pockets. With this understanding, we decouple $\mathcal{H}_{I}$ by introducing the nematic bosonic Hubbard-Stratonovich field $\phi(\mathbf{q})$, and we get the action

$$
S=T \sum_{\mathbf{q}, \omega_{n}}\left(\chi_{\mathrm{nem}}^{0}\left(\mathbf{q}, i \omega_{n}\right)^{-1}-g\right)\left|\phi_{\mathbf{q}, \omega_{n}}\right|^{2}+\cdots
$$

The bare nematic susceptibility $\chi_{\text {nem }}^{0}\left(\mathbf{q}, i \omega_{n}\right)$ is the Fourier transform of $\left\langle T_{\tau} \rho_{B_{1 g}}(\mathbf{q}, \tau) \rho_{B_{1 g}}(\mathbf{q}, 0)\right\rangle_{0}$, where $\langle\cdots\rangle_{0}$ implies average in the BCS ground state with $g=0$.

In Raman spectroscopy of Fe SC the momentum transfer by the photons is typically small compared to the frequency transfer $\left(\omega \gg v_{F} q\right.$, where $v_{F}$ is typical Fermi velocity). Consequently, the $B_{1 g}$ Raman response function probes the imaginary part of the full nematic susceptibility $\chi_{\text {nem }}\left(\mathbf{q}, i \omega_{n}\right)$ in the dynamical limit, and, in random phase approximation, is given by

$$
R_{B_{1 g}}(\omega) \equiv \operatorname{Im} \chi_{\mathrm{nem}}(\omega)=\operatorname{Im}\left(\frac{\chi_{\mathrm{nem}}^{0}(\omega)}{1-g \chi_{\mathrm{nem}}^{0}(\omega)}\right),
$$

where we suppressed the momentum $\mathbf{q}=\mathbf{0}$.

For simplicity we evaluate the above at $T=0$ and by ignoring lifetime broadening of the Bogoliubov quasiparticles. Later we comment about finite- $T$ and finite lifetime effects. Furthermore, we assume a constant superconducting gap $\Delta$ on the electron pockets (for other scenarii see [43, 44]). It is then straightforward to determine the imaginary part of the bare $B_{1 g}$ Raman response 43 .

$$
\operatorname{Im} \chi_{\mathrm{nem}}^{0}(\omega)=\frac{\pi \rho}{2} \frac{(2 \Delta)^{2} \theta\left(\omega^{2}-(2 \Delta)^{2}\right)}{\omega \sqrt{\omega^{2}-(2 \Delta)^{2}}} .
$$

This result is, up to the $d$-wave weighted electron-pocket density of states $\rho=\frac{1}{N} \sum_{\mathbf{k}} \gamma_{B_{1 g}}^{2}(\mathbf{k}) \delta\left(\epsilon_{F}-\epsilon_{\mathbf{k}, e l}\right)$, the usual one for a fully gapped superconductor [45]. The square root divergence for $\omega \rightarrow 2 \Delta$ from above is the well established pair-breaking peak in the electronic Raman spectrum of a superconductor 30. For our considerations we also need the real part of $\chi_{\text {nem }}^{0}(\omega)$ shown in Fig. 1(a). The important aspect of the real part occurs for $|\omega|<2 \Delta$. In this regime it follows after KramersKronig transformation

$$
\operatorname{Re} \chi_{\mathrm{nem}}^{0}(\omega)=\chi_{\mathrm{nem}, \infty}^{0}+\rho \frac{(2 \Delta)^{2} \arcsin \left(\frac{\omega}{2 \Delta}\right)}{\omega \sqrt{(2 \Delta)^{2}-\omega^{2}}} .
$$
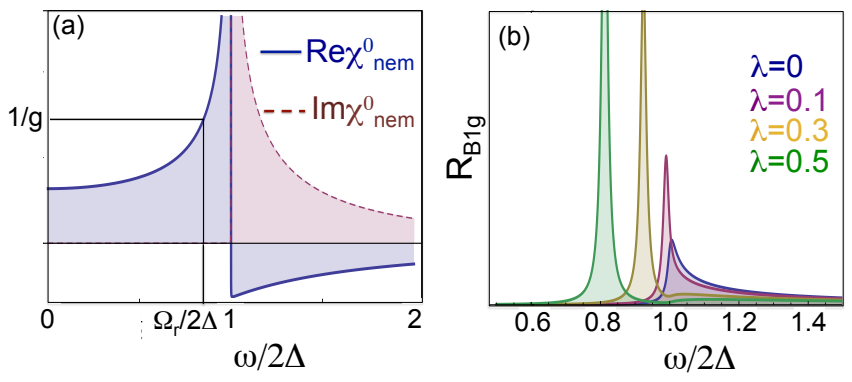

FIG. 1: (a) Real and imaginary parts of the bare BCS Raman response $\chi_{n e m}^{0}$ for an isotropic gap. The interacting response (Eq. 3 in the main text) develops a resonance at $\Omega_{r}<2 \Delta$ when the real part reaches the threshold $1 / g$ (see Eq. 3 in text). (b) Development of the nematic resonance in the superconducting $B_{1 g}$ Raman response $R_{B_{1 g}}$ for different values of the dimensionless coupling constant $\lambda$ (see text). Note that for $\lambda=0.3,0.5$, weak pair breaking continuum are visible.

Besides the low-energy contribution, there is a weakly frequency dependent contribution from the high energy fermions, which we approximate by a constant $\chi_{\text {nem, } \infty}^{0}$. It can be absorbed in the dimensionless coupling $\lambda=$ $\rho g /\left(1-g \chi_{\text {nem, } \infty}^{0}\right)$, and henceforth we take $\lambda$ as the tuning parameter that increases with increasing $g$.

Because of its singular behavior, the leading contribution to $\operatorname{Re} \chi_{\text {nem }}^{0}(\omega)$ for frequencies below $2 \Delta$ is the second term above. If we insert this result into $\mathrm{Eq} 3$, we find a sharp pole in the renormalized Raman response once the condition $1=g \operatorname{Re} \chi_{\text {nem }}^{0}\left(\Omega_{r}\right)$ is fulfilled for $\Omega_{r}<2 \Delta$, where the imaginary part vanishes. As illustrated in Fig.1(a), the square root divergence of $\operatorname{Re} \chi_{\text {nem }}^{0}(\omega)$ as $\omega$ approaches $2 \Delta$ from below, guarantees that the above condition can be satisfied and that a new exciton pole emerges. The resonance frequency of the nematic response is

$$
\Omega_{r}=2 \Delta \sin \theta(\lambda),
$$

where $\theta(\lambda)$ obeys the equation $\sin 2 \theta=2 \lambda \theta$.

In the clean limit and at $T=0 \mathrm{~K}$, the mode is arbitrarily sharp as it occurs below the particle-hole continuum that starts at $2 \Delta$. Near the resonance the electronic Raman response is:

$$
R_{B_{1 g}}(\omega)=Z_{r} \delta\left(\omega-\Omega_{r}\right)
$$

with spectral weight $Z_{r}=\zeta\left(\frac{\Omega_{r}}{2 \Delta}\right)$ determined by $\zeta(x)=$ $\frac{\pi x\left(1-x^{2}\right) \arcsin (x)}{x \sqrt{1-x^{2}}+\left(2 x^{2}-1\right) \arcsin (x)} . Z_{r}$ must be compared with the total weight $\pi^{2} \rho \Delta / 2$ of the usual pair-breaking peak of the BCS theory. Near $2 \Delta$ the spectral weight vanishes linearly $Z_{r} \approx \pi \frac{\Omega_{r}-2 \Delta}{\Delta}$. Since $\rho \Delta \ll 1$, the resonance mode soon acquires a weight comparable to the noninteracting BCS pair-breaking peak. A typical value for $\Omega_{r}=\frac{3}{4} 2 \Delta$ is $Z_{r} \approx 1.45$.

In Fig. 1(b) we show the Raman response for different coupling constants. We included a small but finite in- 
(a)

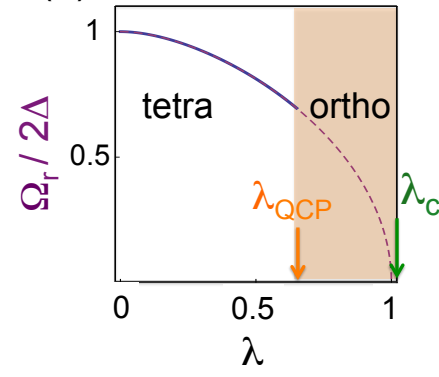

(b)

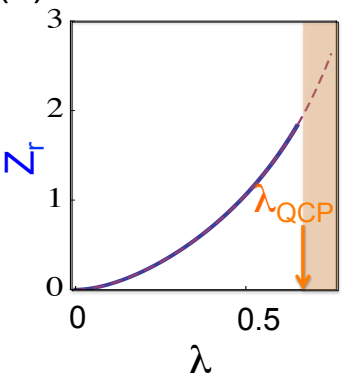

FIG. 2: Evolutions of the nematic resonance energy $\Omega_{r}$ (a) and spectral weight $Z_{r}$ (b) with the dimensionless coupling constant $\lambda$ (dynamic limit). Because of finite nemato-elastic coupling, $Z_{r}$ does not diverge, and $\Omega_{r}$ does not soften completely, at $\lambda_{Q C P}$ (see text).

trinsic width $\Gamma$ that may result from impurity scattering or thermal excitations of quasiparticles [44]. We see that for small nematic coupling constants the pair-breaking peak keeps its line shape but increases in weight. Once $2 \Delta-\Omega_{r}$ is larger than $\Gamma$, a peak that is well separated from the continuum above $2 \Delta$ emerges and sharpens with a Lorentzian line shape for larger coupling strength. The sharpness of the Raman resonance relies heavily on the opening of a full gap in the superconducting spectrum. Nodes of the gap around the electron pockets essentially wash out the nematic resonance [44, making the presence of the nematic resonance a clear indication of the absence of nodes around the electron pockets. We also note that, already in the absence of any nematic correlations, disorder induces a much weaker temperature dependence of the pair-breaking peak energy compared to the simple BCS result for $\Delta(T)$ [46, and we expect a similar effect for the $T$-dependence of the nematic resonance energy $\Omega_{r}$.

Lattice cutoff. - Next we discuss the fate of the nematic resonance as the system approaches the nematic QCP by increasing $\lambda$. From Eq. (2) it is tempting to deduce that the QCP, defined by $g \chi_{\text {nem }}^{0}(0)=1$, is given by $\lambda=1$. This, in turn, would imply that, using Eq. 6, the resonance frequency $\Omega_{r} \rightarrow 0$, while the spectral weight of the resonance $Z_{r} \approx \frac{3 \pi \Delta}{2 \Omega_{r}}$ diverges. However, in practice, this is not the case since, in addition to the purely electronic contribution of Eq. (2), there is a symmetryallowed coupling to the lattice degrees of freedom given by 47 .

$$
H_{c}=\gamma \int d^{d} r \phi(r)\left(\partial_{x} u^{x}-\partial_{y} u^{y}\right)
$$

$\gamma$ is a nemato-elastic coupling constant and $\mathbf{u}$ is the usual phonon displacement field. In the harmonic approximation of the lattice, the effect of the coupling to the phonons is a renormalization of the nematic coupling

constant

$$
g(\mathbf{q}, \omega)=g+\gamma^{2} \frac{\mathbf{q}^{2}}{C_{s}^{0} \mathbf{q}^{2}-\omega^{2}}
$$

$C_{s}^{0}$ is the bare value of the orthorhombic elastic constant and $\mathbf{q}$ is a wave vector along the critical directions of the Brillouin zone [48. Note that, the static limit $g_{\text {stat }} \equiv$ $g(\mathbf{q}, 0)=g+\frac{\gamma^{2}}{C_{s}^{0}}$ and the dynamic limit $g(\mathbf{q}=\mathbf{0}, \omega)=$ $g$ do not commute [49. Thus, the enhancement of the static nematic coupling constant due to the coupling to elastic degrees of freedom does not enter the dynamic Raman response which filters out the purely electronic contribution of the nematic response.

On the other hand, it is the static limit that governs thermodynamics and the actual nematic phase transition. Thus, the condition for the nematic QCP is $\lambda_{\text {stat }}=1$, where $\lambda_{\text {stat }}=\rho g_{\text {stat }} /\left(1-g_{\text {stat }} \chi_{\text {nem }, \infty}^{0}\right)$ is the dimensionless nematic coupling constant in the static regime, renormalized by high energy excitations. Since $g_{\text {stat }}>g$, it follows $\lambda<\lambda_{\text {stat }} \leq 1$. In other words, even at the nematic QCP $\Omega_{r}$ does not soften to zero frequency, and concomitantly, $Z_{r}$ stays finite (see Fig. 2) [50]. In the symmetry-broken nematic phase $\left(\lambda_{\text {stat }}>1\right)$ the behavior of $\left(\Omega_{r}, Z_{r}\right)$ is non-universal, and is beyond the scope of this work.

Raman Experiments. - We now make contact with the experiments on $\mathrm{Fe} \mathrm{SC}$ and in particular with electron doped $\mathrm{BaFe}_{2} \mathrm{As}_{2}$ (Co-Ba122) [16, 19, 20]. We assume that decreasing Co doping $x$ is equivalent to increasing $\lambda$. In the superconducting state of Co-Ba112 the most salient feature of the Raman spectra is a peak observed in $B_{1 g}$ symmetry only [19]. Until now the $B_{1 g}$ peak has been attributed to a $2 \Delta$ BCS pair breaking peak coming from the electron pockets $[19,20,41$. However as first reported in Ref. [20, its Co doping dependance displays a striking behavior which is shown in Fig. 3(a). Coming from the overdoped tetragonal side $(x=0.1$, $\vec{T}_{c}=20 \mathrm{~K}$ ) of the phase diagram, the spectral weight of the peak increases dramatically upon approaching the orthorhombic/nematic phase which occurs below $x=0.065$. It reaches a maximum at $x=0.065$ before collapsing in the orthorhombic phase at lower $x$. As stated above, the spectral weight of a simple BCS Raman pair-breaking peak is expected to scale as the gap energy $\Delta$. However the strong enhancement of $Z_{r}$ around optimal doping, where $T_{c}$ is maximum, cannot be attributed to substantial changes in $\Delta$, since this would imply equivalent changes in $T_{c}$, whereas, in practice the $T_{c}$ changes by less than $20 \%$ within the doping range considered. Moreover the peak energy in units of $\mathrm{k}_{B} \mathrm{~T}_{c}$ actually softens upon approaching $x=0.065$, going from $\sim 5$ to $\sim 4 k_{B} T_{c}$ (see Fig. 3(c)), in a manner consistent with the nematic resonance scenario.

The above observations can be naturally explained by the nematic resonance scenario whose spectral weight $Z_{r}$ 

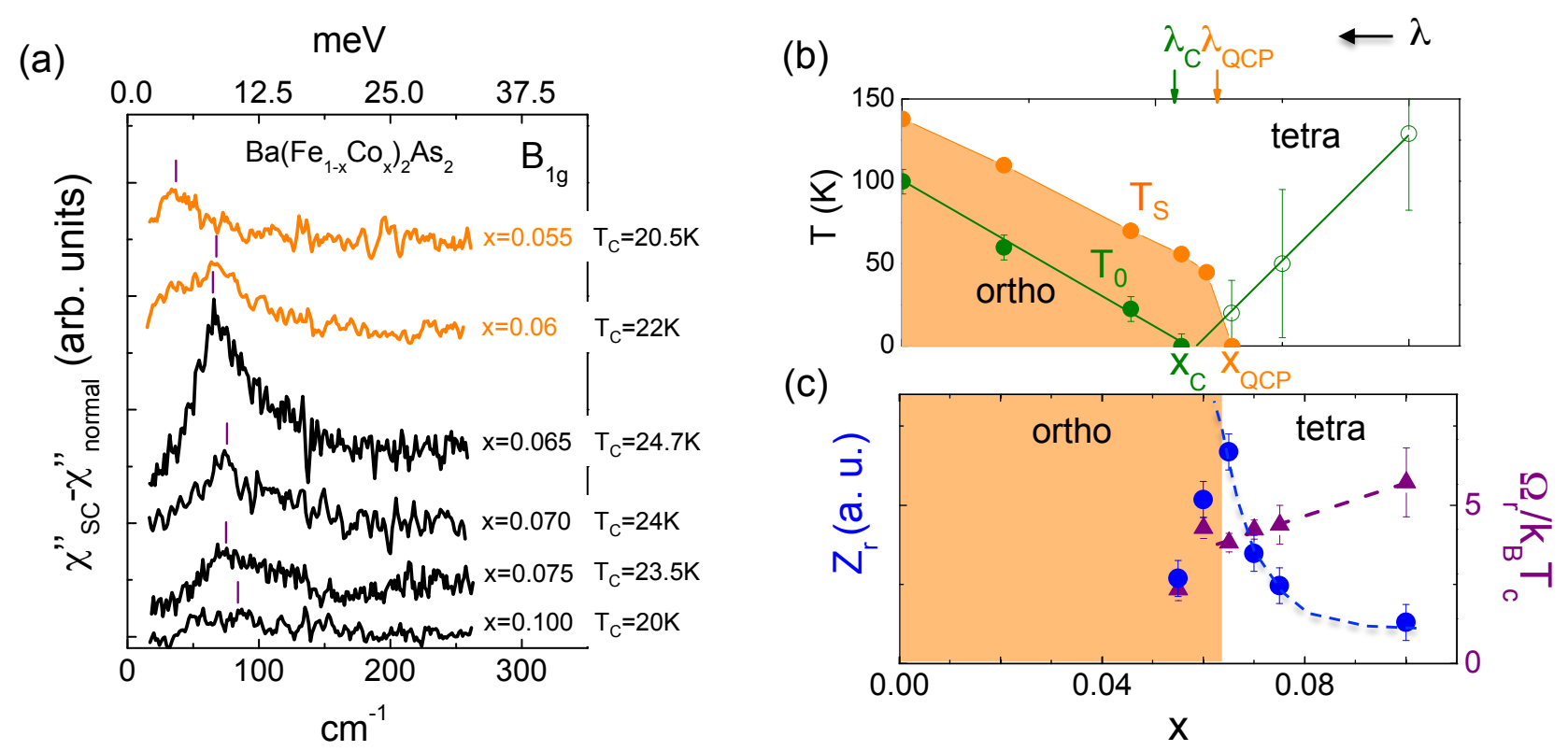

FIG. 3: (a) Co doping $(x)$ dependance of the $B_{1 g}$ Raman response in the superconducting state (SC) of Co-Ba122 20]. The spectra have been subtracted by those just above $T_{c}$ to highlight the SC induced features. $x=0.065$ corresponds to optimal doping $\left(T_{c}=24.7 \mathrm{~K}\right)$ and the spectra in orange correspond to $x$ compositions in the orthorhombic phase. (b) Phase diagram ( $\mathrm{T}, \mathrm{x}$ ) of Co-Ba122 showing the evolution of $T_{S}$ (orange) and of the electronic nematic Curie-Weiss temperature $\left|T_{0}\right|$ (green) deduced from Raman experiments in the normal state 16. Decreasing $x$ content is equivalent as increasing the coupling $\lambda$ of our theory. Note that $T_{0}$ is positive for $x<x_{c}$ (filled symbols) and negative for $x>x_{c}$ (open symbols). (c) Evolution of $Z_{r}$ and $\Omega_{r}$ as a function of Co doping $x$ in Co-Ba122.

is strongly enhanced by the proximity of an nematic instability. In Co-Ba122 the position of the purely electronic nematic instability $x_{c}$ can be determined by looking at the enhancement of Raman $B_{1 g}$ nematic fluctuations observed in the normal state of the tetragonal phase [16. The enhancement of the associated nematic susceptibility was found to follow a Curie-Weiss temperature dependence over a wide range of Co doping from $x=0$ to $x=0.1$. As shown in Fig. 3(b), the extracted CurieWeiss temperature $T_{0}$ is lower than $T_{S}$, the tetragonal to orthorhombic transition temperature, and extrapolates to $\mathrm{T}=0 \mathrm{~K}$ at $x_{c} \sim 0.055$. In a purely electronic model the spectral weight $Z_{r}$ would diverge at $x_{c}$. However, as already stressed above, due to finite nemato-elastic coupling, the actual quantum critical point is moved to a higher doping $x_{Q C P}$, between $x=0.06\left(T_{S}=46 \mathrm{~K}\right)$ and $x=0.065\left(T_{S}=0 \mathrm{~K}\right)$, where $Z_{r}$ and $\Omega_{r}$ are still finite as observed experimentally (Fig. 3(b)) .

We note that a similar divergence of the $B_{1 g}$ peak spectral weight has been observed recently in Co doped $\mathrm{NaFeAs}$ close to the boundary between the tetragonal and orthorhombic phase 21]. There the $B_{1 g}$ resonance was found to be almost Lorentzian below $T_{c}$, with a linewidth significantly smaller than in the case of CoBa122: $1 \mathrm{meV}$ and $5 \mathrm{meV}$ respectively near optimal doping. This difference possibly reflects a smaller coupling constant $\lambda$ in Co-Ba122 which would make the nematic resonance closer to the $2 \Delta$ continuum and there- fore broader. Recent ARPES data in Co-Ba122 indeed indicate that the $B_{1 g}$ peak energy is only slightly below $2 \Delta$ of one of the electron pocket [52]. Disorder may also play a role as optimally doped Co-Ba122 has a significantly higher Co content than Co-Na111 (see supplemental materials for theoretical fits of the experimental lineshapes [51]). However, we stress that, while the lineshape of the resonance can be material dependent, the key feature of our theory, namely the enhancement of the spectral weight near the nematic QCP, is observed experimentally in both systems.

The nematic resonance scenario captures remarkably well the salient features of the $B_{1 g}$ peak observed in the SC state of electron doped Fe SC: its nematic symmetry $\left(B_{1 g}\right)$ and the strong enhancement of its spectral weight near the nematic QCP. It also makes a direct link between the nematic response in the normal and superconducting state via Eq. 3. close to $x_{c}$ the enhanced nematic susceptibility of the normal state converts into a sharp nematic resonance at finite energy in the SC state because of the gap opening.

Furthermore, our theory leads to the following three predictions which can be verified in future experiments. (i) From Eq. 6, and assuming $\lambda$ to be $T$-independent, we expect that $\Omega_{r} \propto \Delta$ upon varying temperature. (ii) Due to the nemato-elastic coupling of Eq. 8 the orthorhombic elastic constant is renormalized to $C_{s}=C_{s}^{0}-\gamma^{2} \chi_{\text {nem }}(\omega=$ $0)$. From Eq. 7 and from Kramers-Kronig relation we de- 
duce that the elastic softening $\delta C_{s} \equiv C_{s}-C_{s}^{0}$ scales as $\delta C_{s} \propto Z_{r} / \Omega_{r}$ upon approaching the nematic transition deep in the SC state, as a function of either doping or temperature. (iii) The nematic resonance will lead to single particle renormalizations (like peak-dip-hump features) everywhere on the electron pockets which should be visible in ARPES experiments.

Conclusion. - In summary we have demonstrated the generic presence of a nematic resonance in the SC state of systems near a nematic quantum critical point like Fe SC. The very existence of this resonance relies only on the presence of a fully gapped Fermi pocket and the proximity of a nematic quantum critical point. Existing Raman data in the $B_{1 g}$ symmetry channel of several Fe SC systems like Co-Ba122 and Co-Na111 indicate that this resonance actually dominates the Raman response in the SC state. It provides the most striking manifestation of nematicity in the superconducting properties of Fe SC.

We are grateful to U. Karahasanovic and R. Hackl for discussions. Y.G. and L.C. acknowledge support from the ANR grant PNICTIDES. J.S. acknowledges support from Deutsche Forschungsgemeinschaft through DFGSPP 1458 Hochtemperatursupraleitung in Eisenpniktiden and thanks the Université Paris Diderot, where this work was done, for its hospitality.

* Electronic address: yann.gallais@univ-paris-diderot.fr

$\dagger$ Electronic address: indranil.paul@univ-paris-diderot.fr

¥ Electronic address: joerg.schmalian@kit.edu

[1] E. Fradkin, S. A. Kivelson, M. J. Lawler, J. P. Eisenstein, and A. P. Mackenzie, Annu. Rev. Condens. Matter Phys. 1, 153 (2010).

[2] R. M. Fernandes, A. V. Chubukov, and J. Schmalian Nature Physics 10, 97 (2014).

[3] R. M. Fernandes, L. H. VanBebber, S. Bhattacharya, P. Chandra, V. Keppens, D. Mandrus, M. A. McGuire, B. C. Sales, A. S. Sefat, and J. Schmalian, Phys. Rev. Lett. 105, 157003 (2010).

[4] M. Yoshizawa, D. Kimura, T. Chiba, S. Simayi, Y. Nakanishi, K. Kihou, C. -H. Lee, A. Iyo, H. Eisaki, M. Nakajima and S. Uchida J. Phys. Soc. Jpn 81, 024604 (2012).

[5] A. E. Böhmer, P. Burger, F. Hardy, T. Wolf, P. Schweiss, R. Fromknecht, M. Reinecker, W. Schranz, C. Meingast, Phys. Rev. Lett. 112, 047001 (2014).

[6] J.-H. Chu, J. G. Analytis, K. De Greve, P. L. McMahon, Z. Islam, Y. Yamamoto, I. R. Fisher, Science 329, 824 (2010).

[7] M. A. Tanatar, E. C. Blomberg, A. Kreyssig, M. G. Kim, N. Ni, A. Thaler, S. L. Bud'ko, P. C. Canfield, A. I. Goldman, I. I. Mazin and R. Prosorov, Phys. Rev. B 81, 184508 (2010).

[8] J.-H. Chu, H.-H. Kuo, J. G. Analytis, I. R. Fisher, Science 337, 710 (2012).

[9] S. Kasahara, H. J. Shi, K. Hashimoto, S. Tonegawa, Y. Mizukami, T. Shibauchi, K. Sugimoto, T. Fukuda, T. Terashima, A. H. Nevidomskyy and Y. Matsuda, Nature
486, 382 (2012).

[10] X. Lu, J. T. Park, R. Zhang, H. Luo, A. H. Nevidomskyy, Q. Si and P. Dai, Science 345, 657 (2014).

[11] A. Dusza, A. Lucarelli, F. Pfuner, J.-H. Chu, I.R. Fisher, L. Degiorgi, Europhys. Lett. 93, 37002 (2011).

[12] I. R. Fisher, L. Degiorgi, Z. X. Shen, Rep. Prog. Phys. 74, 124506 (2011).

[13] T. M. Chuang, M. P. Allan, J. Lee, Y. Xie, N. Ni, S. L. Bud'ko, G. S. Boebinger, P. C. Canfield, J. C. Davis, Science 327, 181 (2010).

[14] M. Yi, D. Hu, J. -H. Chu, J. G. Analytis, A. P. Sorini, A. F. Kemper, B. Moritz, S. -K. Mo, R. G. Moore, M. Hashimoto, W. S. Lee, Z. Hussain, T. P. Devereaux, I. R. Fisher and Z. -X. Shen, Proc. Natl. Acad. Sci. USA 108, 6883 (2011).

[15] E. P. Rosenthal, E. F. Andrade, C. J. Arguello, R. M. Fernandes, L. Y. Ying, X. C. Wang, C. Q. Jin, A. J. Millis and A. N. Pasupathy, Nat. Phys. 10, 225 (2014).

[16] Y. Gallais, R. M. Fernandes, I. Paul, L. Chauvière, Y.-X. Yang, M.-A. Méasson, M. Cazayous, A. Sacuto, D. Colson, and A. Forget, Phys. Rev. Lett. 111, 267001 (2013).

[17] R. M. Fernandes, A. V. Chubukov, J. Knolle, I. Eremin, and J. Schmalian, Phys. Rev. B 85, 024534 (2012).

[18] R. M. Fernandes, S. Maiti, P. Wölfle, A. V. Chubukov, Phys. Rev. Lett. 111, 057001 (2013).

[19] B. Muschler, W. Prestel, R. Hackl, T.P. Devereaux, J.G. Analytis, J.H. Chu and I.R. Fisher, Phys. Rev. B 80, 180510(R) (2009).

[20] L. Chauvière, Y. Gallais, M. Cazayous, M. -A. Méasson, A. Sacuto, D. Colson and A. Forget, Phys. Rev. B 82, 180521 (2010).

[21] V. K. Thorsmolle, M. Khodas, Z. P. Yin, Chenglin Zhang, S. V. Carr, Pengcheng Dai and G. Blumberg, arXiv:1410.6456

[22] J. Rossat-Mignot, L. Regnault, C. Vettier, P. Bourges, P. Burlet, J. Bossy, J. Henry and G. Lapertot, Physica C 185-189, 86 (1991)

[23] V. Hinkov, S. Pailhès, P. Bourges, Y. Sidis, A. Ivanov, A. Kulakov, C. T. Lin, D. P. Chen, C. Bernhard and B. Keimer, Nature 430, 650 (2004)

[24] P. Bourges, B. Keimer, S. Pailhès, L. P. Regnault, Y. Sidis and C. Ulrich, Physica C 424, 45 (2005)

[25] A. D. Christianson, E. A. Goremychkin, R. Osborn, S. Rosenkranz, M. D. Lumdsen, C. D. Malliakas, I. S. Todorov, H. Claus, D. Y. Chung, M. G. Kanatzidis, R. I. Bewley and T. Guidi, Nature 456, 930 (2008)

[26] M. D. Lumsden, A. D. Christianson, D. Parshall, M. B. Stone, S. E. Nagler, G. J. McDougall, H. A. Mook, K. Lokshin, T. Egami, D. L. Abernathy, E. A. Goremychkin, R. Osborn, M. A. McGuire, A. S. Sefat, R. Jin, B. C. Sales and D. Mandrus, Phys. Rev. Lett. 102, 107005 (2009)

[27] D. Inosov, J. T. Park, P. Bourges, D. L. Sun, Y. Sidis, A. Schneidewind, K. Hradil, D. Haug, C. T. Lin, B. Keimer and V. Hinkov, Nature Phys. 6, 178 (2010)

[28] Ar. Abanov and A. V. Chubukov Phys. Rev. Lett. 83, 1652 (1999).

[29] M. Eschrig and M. R. Norman, Phys. Rev. Lett. 85, 3261 (2000)

[30] T. P. Devereaux and R. Hackl, Rev. Mod. Phys. 79, 175 (2007)

[31] C. Fang, H. Yao, W.-F. Tsai, J.-P. Hu, and S.A. Kivelson, Phys. Rev. B 77, 224509 (2008).

[32] C. Xu, M. Muller and S. Sachdev, Phys. Rev. B 78, 
020501 (2008).

[33] J. Kang and Z. Tesanovic, Phys. Rev. B 83, 020505 (2011)

[34] I. Paul, Phys. Rev. Lett. 107, 047004 (2011).

[35] C.C. Lee, W.-G. Yin and W. Ku, Phys. Rev. Lett. 103, 267001 (2009).

[36] F. Krüger, S. Kumar, J. Zaanen and J. van den Brink, Phys. Rev. B 79, 054504 (2009).

[37] W. Lv, J. Wu and P. Phillips, Phys. Rev. B 80, 224506 (2009), W. Lv, F. Krüger and P. Phillips, Phys. Rev. B 82, 045125 (2010).

[38] C. -C. Chen, J. Maciejko, A. P. Sorini, B. Moritz, R. R. P. Singh and T. P. Devereaux, Phys. Rev. B 82, 100504 (2010).

[39] S. Onari and H. Kontani, Phys. Rev. Lett. 109, 137001 (2012).

[40] H. Zhai, F. Wang, D. -H. Lee, Phys. Rev. B 80, 064517 (2009)

[41] I.I. Mazin, T.P. Devereaux, J.G. Analytis, J.-H. Chu, I.R. Fisher, B. Muschler and R. Hackl, Phys. Rev. B 82, 180502R (2010).

[42] B. Valenzuela, M. J. Calderòn, G. Léon and E. Bascones, Phys. Rev. B 87, 075136 (2013).

[43] G. R. Boyd, T. P. Devereaux, P. J. Hirschfeld, V. Mishra, and D. J. Scalapino, Phys. Rev. B 79, 174521 (2009).
[44] $\Gamma=0.02 \Delta$ was used for 1 (b). See Supplemental Material, which includes ref. [53], for the case of nodal and anisotropic gaps and for calculations of the Raman response with a larger lifetime broadening.

[45] M. V. Klein and S. B. Dierker, Phys. Rev. B 29, 4976 (1984).

[46] T. P. Devereaux, Phys. Rev. B 47 5230, (1993)

[47] We use a unit cell with one Fe atom. If one uses two Fe atoms per unit cell the $B_{1 g}$ symmetry becomes the $B_{2 g}$ symmetry and $\phi$ couple to $\partial_{x} u^{y}+\partial_{y} u^{x}$.

[48] A. Cano, M. Civelli, I. Eremin and I. Paul, Phys. Rev. B 82 020408(R) (2010).

[49] H. Kontani, Y. Yamakawa, Phys. Rev. Lett. 113, 047001 (2014).

[50] If one allows for a small but finite photon momentum, the elastic contribution is still suppressed, albeit not completely, and $\lambda<\lambda_{\text {stat }}$ still holds.

[51] See Supplemental Material for theoretical fits of the $\mathrm{B}_{1 g}$ experimental spectra in Co-Ba122 and Co-Na111.

[52] T. Hajiri, T. Ito, M. Matsunami, B. H. Min, Y. S. Kwon and S. Kimura, J. Phys. Soc. Japan 83, 093703 (2014)

[53] G. R. Boyd, P. J. Hirschfeld and T. P. Devereaux, Phys. Rev. B 82, 134506 (2010) 